\title{
High-Resolution ISAR Imaging Based on Improved Sparse Signal Recovery Algorithm
}

\author{
Junjie Feng $\mathbb{D},{ }^{1}$ Yinan Sun, ${ }^{2}$ and XiuXia Ji $\mathbb{C}^{3}$ \\ ${ }^{1}$ School of Physics and Electrical Engineering, Liupanshui Normal University, Liupanshui, Guizhou, China \\ ${ }^{2}$ Wuhan University, School Electronic Information, Wuhan 430072, China \\ ${ }^{3}$ Nanjing Vocational College of Information Technology, Nanjing 210023, China
}

Correspondence should be addressed to Junjie Feng; fhzy0929@163.com

Received 31 January 2021; Revised 1 March 2021; Accepted 20 March 2021; Published 2 April 2021

Academic Editor: Liangtian Wan

Copyright (c) 2021 Junjie Feng et al. This is an open access article distributed under the Creative Commons Attribution License, which permits unrestricted use, distribution, and reproduction in any medium, provided the original work is properly cited.

In order to solve the problem of high-resolution ISAR imaging under the condition of finite pulses, an improved smoothed L0 norm (SL0) sparse signal reconstruction ISAR imaging algorithm is proposed. Firstly, the ISAR imaging is transformed into the optimization problem of minimum L0 norm. Secondly, a single-loop structure is used instead of two loop layers in SL0 algorithm which increases the searching density of variable parameter to ensure the recovery accuracy. Finally, the compared step is added to ensure the optimization solution along the steepest descent gradient direction. The experimental results show that the proposed algorithm has better imaging effect.

\section{Introduction}

Inverse synthetic-aperture radar (ISAR) has the characteristics of all-weather, all-time, long-range, and high-resolution which is widely used in military and civil fields, such as to detect, locate, track, and estimate the parameters of the target [1-4]. The range and azimuth resolution of ISAR imaging are related to radar system bandwidth and imaging accumulation angle, respectively. The range resolution is usually improved by increasing the bandwidth of the transmitted signal, and the azimuth resolution is improved by increasing the observation angle range. In a long observation time, the scattering characteristics of noncooperative moving targets change greatly, and there may be a large Doppler time-varying, which is not conducive to imaging and makes it difficult to improve the azimuth resolution. So implementing imaging in a short time duration is meaningful.

In recent years, compressive sensing (CS) has become very popular in signal processing [5-8]. It is a new signal processing theory proposed by Donoho which is developed from the theory of signal sparse decomposition and approximation. The theory shows that as long as the signal is compress- ible or sparse in a certain transform domain, the original signal can be reconstructed with a small number of sampled signals. It is a technique proposed to improve signal separation ability using a prior sparse property information of the signal.

In radar imaging, the targets are generally composed of strong scattering points, which are sparsely distributed in the imaging region. So radar signal processing based on compressive sensing theory has been widely studied [912], such as SAR imaging [13], ISAR imaging [14, 15], and MIMO radar imaging [16]. The CS theory is not sensitive to data loss, which can effectively improve the imaging problem of radar imaging system in the case of data loss and improve the signal processing ability. The Bayesian sparse signal reconstruction ISAR imaging algorithm based on hierarchical prior model correlation prior is proposed in [17], which can obtain better focusing effect in noisy environment. However, the algorithm is computational complexity. By using the sparsity of ISAR target in azimuth direction, the target image can be obtained by solving the L1 norm minimization in [18]. Combination of local sparsity constraint and nonlocal total variation is discussed in 
[19]. A two-dimensional structure mode-coupled Bayesian (PC-SBL) ISAR imaging algorithm is proposed in [20], which can realize high-resolution ISAR imaging, but the algorithm has a large amount of computation, which is not conducive to real-time imaging.

Mohimani et al. proposed the smoothed L0 norm sparse signal recovery algorithm by using the continuous Gaussian function sequence with parameters as smoothing function to approximate the minimum L0 norm [21]. The steepest descent method and gradient projection principle by a double-layer loop is used to obtain the optimal solution. The outer layer establishes a sequence from large to small, and the inner layer uses the steepest descent method to obtain the approximate solution. The reconstruction speed of the algorithm is 2-3 times faster than basis pursuit algorithm when it has the same accuracy.

In order to achieve high-resolution ISAR imaging under the condition of finite pulses, an ISAR imaging algorithm with single-loop structure compared to SL0 sparse signal recovery algorithm is proposed. The imaging problem is transformed to a minimum L0 norm optimization problem to achieve high-resolution ISAR imaging. The steepest descent method should reduce the cost function at every step, but it is not really along the descending direction in the actual solving process. Therefore, in the above algorithm, the step of checking whether to descend is added in each iteration. If not, the midpoint of the previous point and the current point is taken for correction to ensure that the search direction is along the steepest descent direction. By using the improved algorithm, ISAR imaging is more intensive with limited pulse numbers. Real data ISAR images obtained using the proposed method is competitive to the several popular methods.

The paper is organized as follows. "ISAR Imaging Model" introduces the ISAR sparse imaging model. In "ISAR Imaging Algorithm," the proposed reconstruction algorithm is introduced in detail. Simulation and real data ISAR imaging results are presented in "Experimental Results." Finally, "Conclusion" provides the conclusion.

\section{ISAR Imaging Model}

Assuming that the target is located in far field, the radar transmits linear frequency modulization signal as

$$
y(t)=\exp \left(j 2 \pi\left(f_{0} t+\frac{1}{2} \mu_{0} t^{2}\right)\right) t \in\left[-\frac{T}{2}, \frac{T}{2}\right],
$$

where $f_{0}$ is the center frequency, $\mu_{0}$ is the slope, and $T$ is the pulse period. Then, the complex echo signal of scattering point $P(x, y)$ is

$$
y(t)=\sigma e^{-j 2 \pi f_{0} \tau(t)+j \pi \mu_{0}(t-\tau(t))^{2}},
$$

where $\tau(t)$ is time delay and $\sigma$ is signal amplitude. After range compression, the signal can be expressed as

$$
y(t)=\sigma \exp \left(-j 2 \pi f_{0} \tau(t)\right) \sin c\left(\mu_{0} T(t-\tau(t))\right) .
$$

After the envelope compensation, the target rotates around the coordinate axis at a constant angular velocity $\omega$. Assuming the initial position of the scattering point is $\left(x_{0}, y_{0}\right)$, the time delay is mainly determined by $x_{0} \sin (\omega$ $t)+y_{0} \cos (\omega t)$. After the Taylor expansion, $x_{0} \sin (\omega t)+$ $y_{0} \cos (\omega t)$ is expressed as

$$
x_{0} \sin (\omega t)+y_{0} \cos (\omega t) \approx y_{0}+x_{0} \omega t \text {. }
$$

Then, $\tau(t) \approx \tau_{0}+\left(2\left(y_{0}+x_{0} \omega t\right) / c\right)$, where $c$ is light speed. The echo signal of range unite $\tau_{0}+\left(2 y_{0} / c\right)$ is

$$
y(t)=\sigma e^{-\frac{-j 4 \pi x_{0} \omega t}{\lambda}}
$$

where $\lambda=c / f_{0}$ is the wavelength. After discretization, the above formula can be written as

$$
y(n)=\sigma \alpha(n)
$$

where $\alpha(n)=e^{j 2 \pi f_{d} n}, f_{d}=-2 x_{0} \omega d / \lambda$, and $d_{t}$ is the sampling interval. The received signal is written as a matrix; then

$$
y=\Phi x+n
$$

where $x$ is the vector composed of scattering coefficient $\sigma$ and $\Phi$ is sparse matrix.

To solve $x$, the following sparse optimization strategy is used:

$$
\begin{gathered}
\min _{x}\|x\|_{0} \\
\text { s.t. }\|y-\Phi x\|_{2}<\tau,
\end{gathered}
$$

where $\tau$ is a small constant which is related to the noise variance. The ISAR imaging problem is transformed into the reconstruction problem of formula (8).

\section{ISAR Imaging Algorithm}

To solve $x$, the smoothed L0 norm algorithm is proposed in [21]. The Gauss sequence $F_{\sigma}(x)=\sum_{i} \exp \left(-x_{i}^{2} / \sigma\right)$ is used to approach the L0 norm. The minimum L0 norm can be obtained by gradually reducing the value of control parameter $\sigma$. A two-layer method was proposed to solve the sparse signal recovery problem in the algorithm. In order to further improve the reconstruction effect of sparse signal, the one-order negative exponential function sequence $G_{\sigma}(x)=\sum_{i} \exp \left(-\left|x_{i}\right| / \sigma\right)$ is proposed as smoothed function to approach L0 norm in the paper. When $\sigma$ $\longrightarrow \infty, G_{\sigma}(x)$ approximates L1 norm. When $\sigma \longrightarrow 0, G_{\sigma}$ $(x)$ approximates L0 norm. Therefore, the sparse solution can be searched with high probability at the beginning of iteration.

In the double-loop structure of SL0 algorithm, the solution of $\sigma=\sigma_{j}$ is only as the initial value of $\sigma=\sigma_{j+1}$, so it is not necessary to solve the exact solution of $\sigma=\sigma_{j}$ in the inner loop. Therefore, the sparse signal reconstruction algorithm in this paper cancels the inner loop step and 


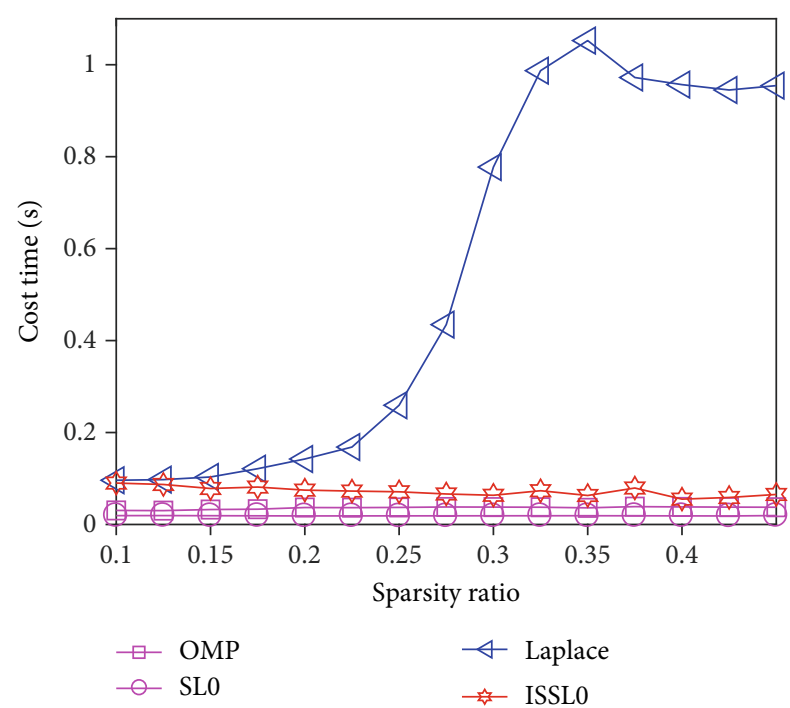

FIgURE 1: Computation costs of different algorithms.

adopts the single loop instead of the double-loop structure in SLO algorithm [52], the interval between control parameters $\sigma_{j}$ and $\sigma_{j+1}$ is reduced, only one gradient descent method is used to solve the minimum value of the cost function, and then the solution of $x$ is given as the initial value of $\sigma=\sigma_{j+1}$. Due to the solution obtained by the steepest descent method is not necessarily in the feasible solution region, it is necessary to project the solution obtained by each iteration into the feasible solution region using the projection method. The proposed algorithm is called the improved single-loop smoothed L0 norm (ISSL0) algorithm. The ISSL0 algorithm can be expressed as follows:

Initialization is as follows:

(1) Let $\widehat{x}_{0}$ be equal to the minimum L2 norm solution of $y=\Phi x$, obtained by $\widehat{x}_{0}=\Phi^{H}\left(\Phi \Phi^{H}\right)^{-1} y$

(2) Choose a suitable decreasing sequence for $\{\sigma\},\left[\sigma_{1}\right.$, $\left.\sigma_{2}, \cdots, \sigma_{J}\right]$

for $j=1, \cdots, L$

(i) Let $\sigma=\sigma_{j}$ and $\beta=L-l+1 / L$

(ii) Minimize the function $G_{\sigma}(x)$ on the feasible set $x$ $=\{x:|\Phi x-y|<\varepsilon\}$

(iii) Let $\delta$ be the gradient of $G_{\sigma}(x)$

(iv) For $x$, let $x_{1} \longleftarrow x-\mu \sigma \delta$

(v) If $\left|\Phi x_{1}-y\right|>\varepsilon$, project $x$ back into the feasible set:

$$
x_{1} \longleftarrow x_{1}+\Phi^{H}\left(\Phi \Phi^{H}\right)^{-1}\left(y-\Phi x_{1}\right)
$$

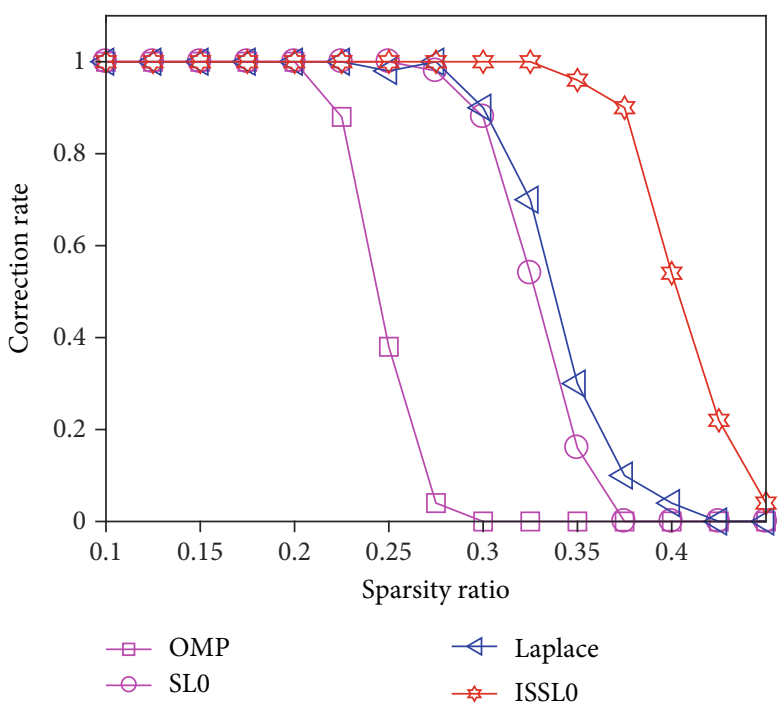

FIgure 2: Correct position estimation of different algorithms.

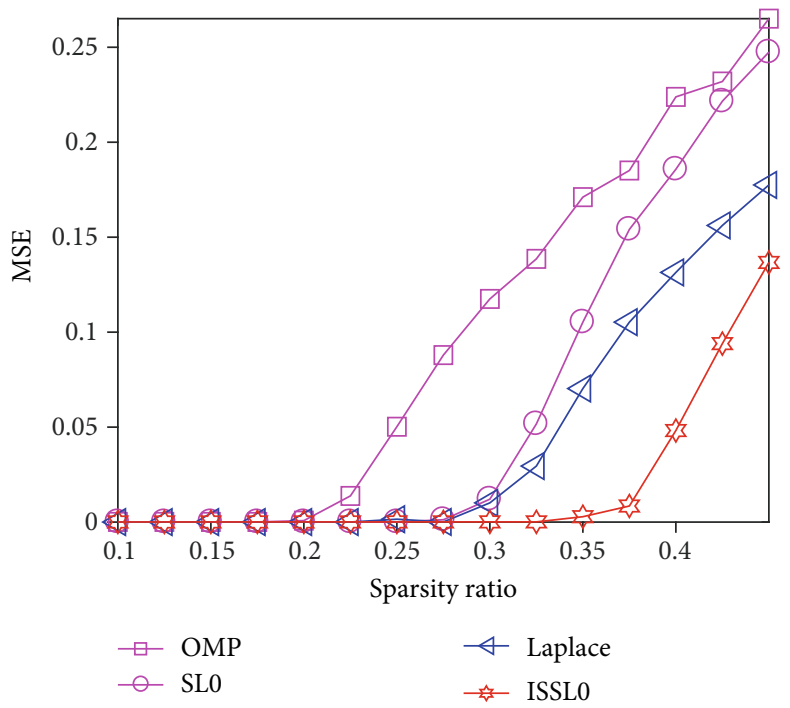

Figure 3: MSE of different algorithms.

While $G_{\sigma}\left(x_{1}\right)>G_{\sigma}(x)$

$$
x_{2}=\left(x+x_{1}\right) / 2
$$

update $G_{\sigma}\left(x_{2}\right)$

If $G_{\sigma}\left(x_{2}\right)<G_{\sigma}(x)$

$$
x=x_{2}
$$

(vi) Compare step

(3) Final solution is $x=\widehat{x}_{J}$. 


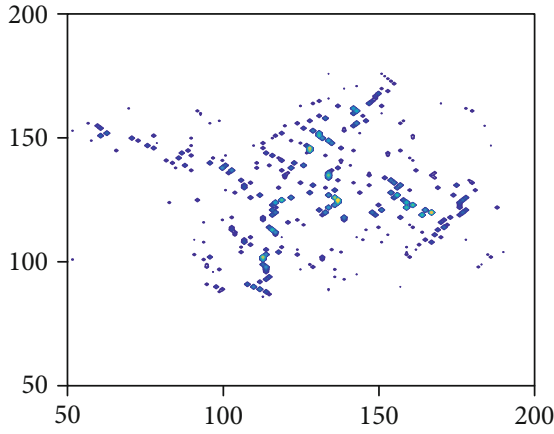

(a)

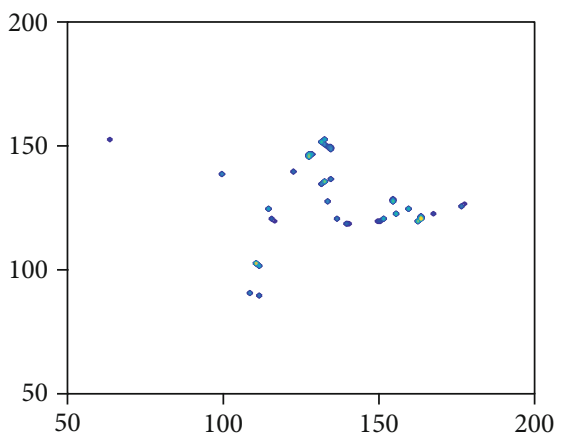

(c)

FIGURE 4: ISAR images using 16 pulses.

The steepest descent method should reduce the cost function at every step, but it is not the descending direction in the actual solution process. Therefore, in the above algorithm, the step of checking whether to descend is added in the process of each iteration. If not, the midpoint of the previous point and the current point are taken for correction to ensure that the search direction is along the steepest descent direction. By using the single loop and compared step, the algorithm ensures the reconstruction accuracy and the computation amount cannot increase. The choice of step size factor in the steepest descent method is important. For a large step size, it may not converge, but for a very small step size, the computation efficiency is low. The step size should decrease when the searching point approaches the minimum solution. For search step factor, because the search point is far away from the minimum value point at the initial value, a larger step size is selected. When the search point is gradually close to the minimum value, the search step size should be gradually reduced. Regulatory factors are used to adjust the step; let $\mu=\beta(\max (|x|) / L)$.

\section{Experimental Results}

4.1. Simulation 1: One-Dimensional Sparse Signal Recovery. In order to verify the reconstruction performance of the algorithm, the paper tests the algorithm through the MATLAB processing platform. The signal model with noise is $y=\Phi x+n$, where $\Phi$ is constructed by selecting

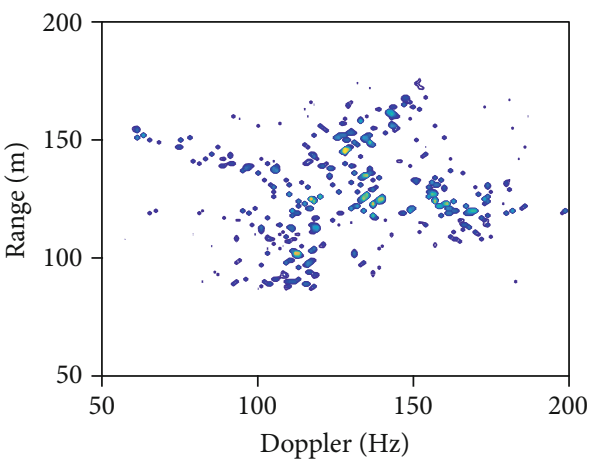

(b)

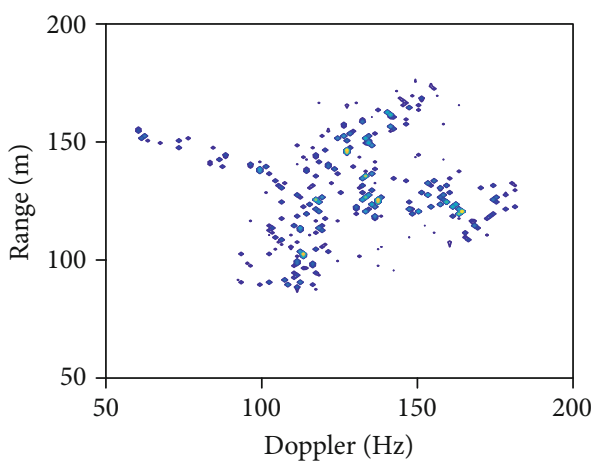

(d)

(a) OMP. (b) Laplace. (c) SL0. (d) ISSL0.

its components from $N(0,1)$ and $x$ is the sparse signal, the nonzero elements of sparse signal consist of random signal \pm 1 . For the SL0 method, the numbers of outer loop and inner loop are 20 and 10, respectively. For the ISSL0 algorithm, the loop number is 200 . For the ISSL0 algorithm, the loop number is 200 . The experiment was implemented 100 times. The MSE is defined as $(1 / N)\|x-x \wedge\|^{2}$. Changing the sparsity ratio of the signal, the curve of reconstruction time, correction rate, and MSE for different algorithms of sparse signal are shown in Figures 1-3. As can be seen from Figure 1, the ISSL0 algorithm takes more time than the OMP [22] algorithm and SLO algorithm and shorter than the Laplace [23] algorithm. From Figures 2 and 3 , we can see that the performances of ISSL0 algorithm are competitive with other algorithms.

4.2. Simulation 2: ISAR Imaging Using Real Data. A set of real data of the Yak-42 plane is used to demonstrate the performance of the proposed ISAR imaging algorithm. The related parameter descriptions of the radar data are listed as follows: the carrier frequency is $10 \mathrm{GHz}$ with signal bandwidth of $400 \mathrm{MHz}$, and the range resolution is $0.375 \mathrm{~m}$. The center carrier frequency is $5.52 \mathrm{GHz}$, and the pulse repetition frequency is $50 \mathrm{~Hz}$. 256 echo pulses are selected as experimental data. 16, 32, and 64 pulses are implemented. For the SL0 method, the numbers of outer loop and inner loop are 20 and 10, respectively. For the ISSL0 algorithm, the loop number is 200 . The simulation results are compared visually and 


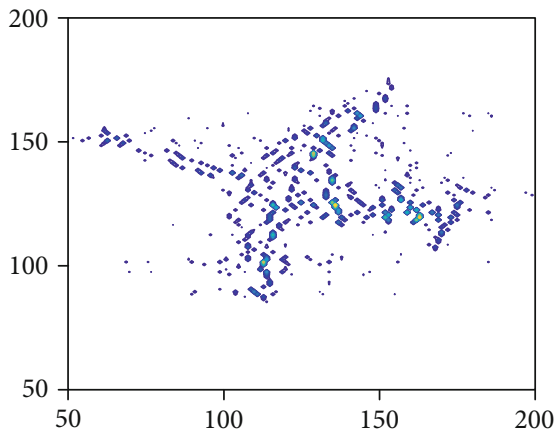

(a)

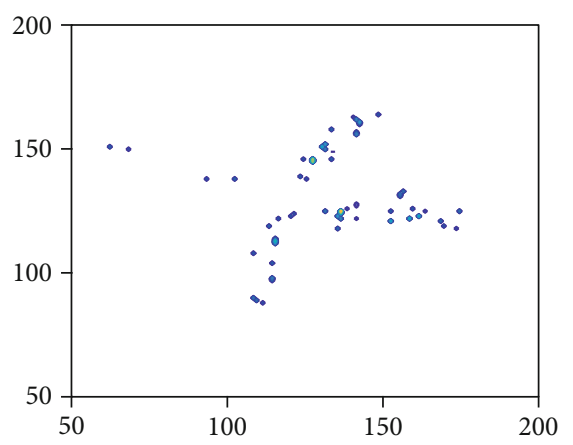

(c)

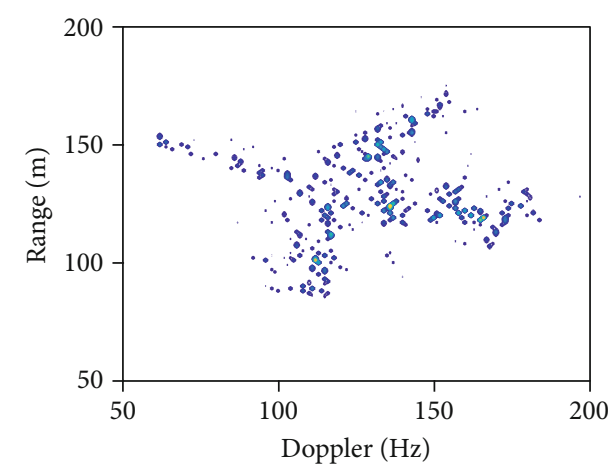

(b)

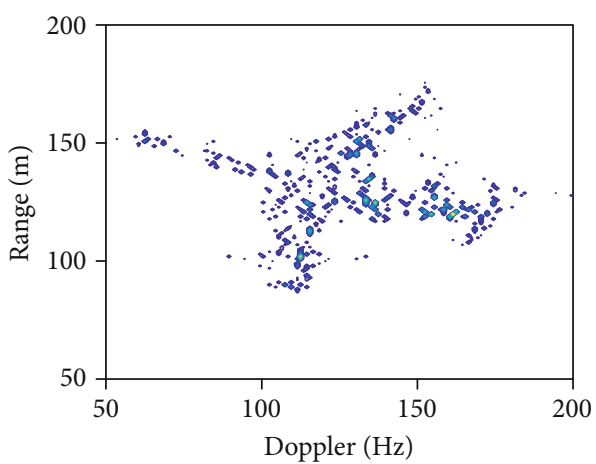

(d)

FIgURE 5: ISAR images using 32 pulses. (a) OMP. (b) Laplace. (c) SL0. (d) ISSL0.

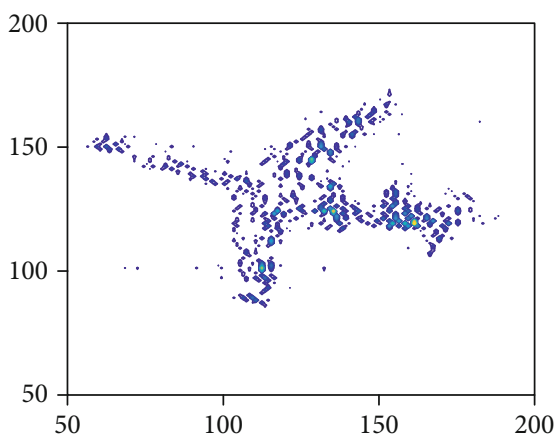

(a)

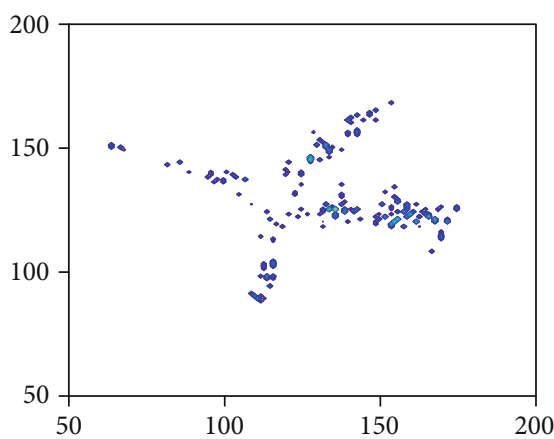

(c)

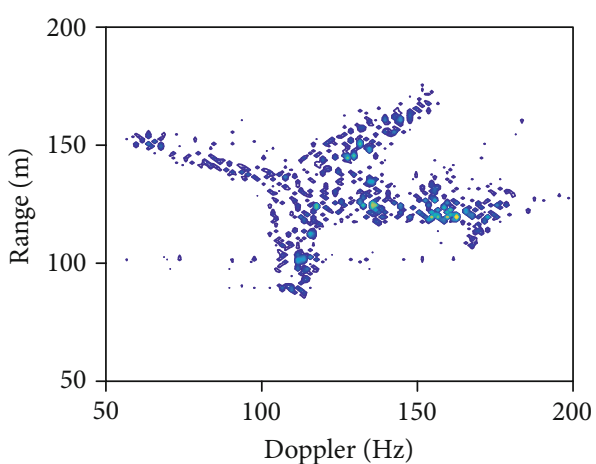

(b)

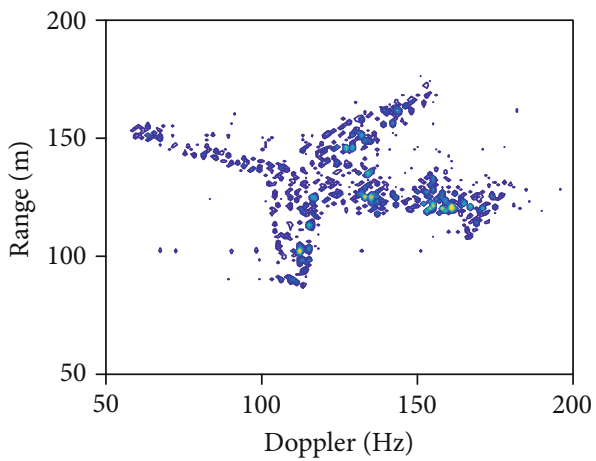

(d)

FIgURE 6: ISAR images using 64 pulses. (a) OMP. (b) Laplace. (c) SL0. (d) ISSL0. 
quantitatively to those images obtained by OMP, Laplace, SL0, and ISSL0 algorithms in Figures 4-6. It is noticeable that more amounts of pulses generally lead to better imagery results. When the number of pulses is 64, the four sparse signal reconstruction algorithms are better. For the SL0 algorithm, when 16 and 32 pulses are used, some information of scattered points is missing. When the number of pulses is small, a large number of false scattering points appear in the OMP and Laplace algorithms. Regardless of the number of pulses, the ISSL0 algorithm keeps the aircraft in a good shape without false scattered points, and the geometric representation of the target is clear.

\section{Conclusion}

One improved sparse signal recovery ISAR imaging algorithm is proposed to ISAR imaging. A single-loop structure is proposed to solve the optimal solution. The revised step is added to ensure the searching direction is decrease compared to the traditional smoothed L0 norm recovery algorithm. The experiment results verify that the proposed algorithm can improve ISAR imaging quality.

\section{Data Availability}

All data sources are reliable and the corresponding author can be contacted.

\section{Conflicts of Interest}

The author declares no conflicts of interest.

\section{Acknowledgments}

This work was partially supported by the National Science Foundation of China (no. 61471191) and the Natural Science Research Project of Jiangsu Colleges and Universities (19KJB510043).

\section{References}

[1] D. H. Kim, D. K. Seo, and H. T. Kim, "Efficient classification of ISAR images," IEEE Transaction on Antennas and Propagation, vol. 53, no. 5, pp. 1611-1621, 2005.

[2] L. Zhang, M. Xing, C.-W. Qiu, J. Li, and Z. Bao, “Achieving higher resolution ISAR imaging with limited pulses via compressed sampling," IEEE Geoscience and Remote Sensing Letters, vol. 6, no. 3, pp. 567-571, 2009.

[3] H. Wang, Y. Quan, M. Xing, and S. Zhang, "ISAR imaging via sparse probing frequencies," IEEE Geoscience and Remote Sensing Letters, vol. 8, no. 3, pp. 451-455, 2011.

[4] Y. Chen, Q. Zhang, N. Yuan, Y. Luo, and H. Lou, "An adaptive ISAR-imaging-considered task scheduling algorithm for multi-function phased array radars," IEEE Transactions on Image Processing, vol. 63, no. 19, pp. 5096-5110, 2015.

[5] R. Chartrand, "Exact reconstruction of sparse signals via nonconvex minimization," IEEE Signal Processing Letters, vol. 14, no. 10, pp. 707-710, 2007.

[6] D. Needell and R. Vershynin, "Uniform uncertainty principle and signal recovery via regularized orthogonal matching pursuit," Foundations of Computational Mathematics, vol. 9, no. 3, pp. 317-334, 2009.

[7] T. Wimalajeewa, H. Chen, and P. K. Varshney, "Performance limits of compressive sensing based signal classification," IEEE Transactions on Signal Processing, vol. 60, no. 6, pp. 27582770, 2012.

[8] R. G. Baraniuk, V. Cevher, M. F. Duarte, and C. Hegde, "Model-based compressive sensing," IEEE Transactions on Information Theory, vol. 56, no. 4, pp. 1982-2001, 2010.

[9] L. Wan, K. Liu, Y.-C. Liang, and T. Zhu, "DOA and polarization estimation for non-circular signals in 3-D millimeter wave polarized massive MIMO systems," IEEE Transactions on Wireless Communications, vol. 8, p. 1, 2021.

[10] L. Wan, L. Sun, K. Liu, X. Wang, Q. Lin, and T. Zhu, “Autonomous vehicle source enumeration exploiting non-cooperative UAV in software defined internet of vehicles," IEEE Transactions on Intelligent Transportation Systems, vol. 9, pp. 1-13, 2020.

[11] L. Sun, L. T. Wan, and X. P. Wang, "Learning-based resource allocation strategy for industrial IoT in UAV-enabled MEC systems," IEEE Transactions on Industrial Informatics, vol. 9, p. 1, 2020.

[12] J. Lv, L. Huang, Y. Shi, and X. Fu, "Inverse synthetic aperture radar imaging via modified smoothed $L_{0}$ norm," IEEE Antennas and Wireless Propagation Letters, vol. 13, pp. 1235-1238, 2014.

[13] C. He, L. Liu, L. Xu, M. Liu, and M. Liao, "Learning based compressed sensing for SAR image super-resolution," IEEE Journal of Selected Topics in Applied Earth Observations and Remote Sensing, vol. 5, no. 4, pp. 1272-1281, 2012.

[14] J. Liu, X. Li, S. Xu, and Z. Zhuang, "ISAR imaging of nonuniform rotation targets with limited pulses via compressed sensing," Progress In Electromagnetics Research B, vol. 41, pp. 285-305, 2012.

[15] J. J. Feng, "ISAR imaging based on multiple measurement vector model sparse signal recovery algorithm," Mathematical Problems in Engineering, vol. 2020, Article ID 17435593, 8 pages, 2020.

[16] W. J. Zhang and A. Hoorfar, "A generalized approach for SAR and MIMO radar imaging of building interior targets with compressive sensing," IEEE Antennas and Wireless Propagation Letters, vol. 14, pp. 1052-1055, 2015.

[17] L. F. Zhao, L. Wang, G. Bi, and L. Yang, “An autofocus technique for high-resolution inverse synthetic aperture radar imagery," IEEE Transactions on Geoscience and Remote Sensing, vol. 52, no. 10, pp. 6392-6403, 2014.

[18] X. Zhang, T. Bai, H. Meng, and J. Chen, "Compressive sensingbased ISAR imaging via the combination of the sparsity and nonlocal total variation," IEEE Geoscience and Remote Sensing Letters, vol. 11, no. 5, pp. 990-994, 2014.

[19] L. Zhang, Z.-J. Qiao, M.-D. Xing, J.-L. Sheng, R. Guo, and Z. Bao, "High-Resolution ISAR imaging by exploiting sparse apertures," IEEE Transactions on Antennas and Propagation, vol. 60, no. 2, pp. 997-1008, 2012.

[20] H. Duan, L. Zhang, J. Fang, L. Huang, and H. Li, "Patterncoupled sparse Bayesian learning for inverse synthetic aperture radar imaging," IEEE Signal Processing Letters, vol. 22, no. 11, pp. 1995-1999, 2015.

[21] H. Mohimani, M. Babaie-Zadeh, and C. Jutten, "A fast approach for overcomplete sparse decomposition based on 
smoothed 10 norm," IEEE Transactions on Signal Processing, vol. 57, no. 1, pp. 289-301, 2009.

[22] J. F. Determe, J. Louveaux, L. Jacques, and F. Horlin, "Improving the correlation lower bound for simultaneous orthogonal matching pursuit," IEEE Signal Processing Letters, vol. 3, no. 11, pp. 1642-1646, 2016.

[23] S. D. Babacan, R. Molina, and A. K. Katsaggelos, "Bayesian compressive sensing using Laplace priors," IEEE Transactions on Image Processing, vol. 19, no. 1, pp. 53-63, 2010. 